\title{
Quantification of hand grasp force using a pressure mapping system
}

\author{
Jon W. Nicholas ${ }^{\mathrm{a},{ }^{*}}$, Russel J. Corvese ${ }^{\mathrm{a}}$, Charles Woolley ${ }^{\mathrm{b}}$, and Thomas J. Armstrong ${ }^{\mathrm{b}}$ \\ ${ }^{a}$ Biomedical Engineering, University of Michigan, 2200 Bonisteel Blvd., Ann Arbor, MI 48109-2099, United \\ States \\ ${ }^{\mathrm{b}}$ Center for Ergonomics, Industrial and Operations Engineering, University of Michigan, 1205 Beal Ave., Ann \\ Arbor, MI 48109-2117, United States
}

\begin{abstract}
The goal of this study was to use a pressure sensor to measure the force distribution and contact area of the hand when gripping, pushing, and pulling a cylinder. Data was collected from 10 subjects with no hand impairments and from 1 subject with rheumatoid arthritis (RA). Subjects grasped an aluminum cylinder wrapped with a Tekscan pressure sensor and performed each trial at $25 \%, 50 \%$, and $100 \%$ maximum voluntary exertion. A relationship was found between increasing exertion and increasing hand area with increasing hand contact area. The force distribution maps showed the thenar region of the hand exerts the most force during pushing while the metacarpal joint line exerts the highest force during pulling. The third and fourth phalange were found to exert the highest phalange force during gripping. The force distribution maps from the RA subject showed higher thumb forces and distal phalange forces, relative to the entire phalange, compared to the non-impaired subjects. This suggests that the RA subject compensates for the lack of phalange function with the regions of the hand that still function. Future studies should sample individuals with a larger hand area range and sample more individuals with RA.
\end{abstract}

Keywords: hand, grasp, Tekscan, contact area, rheumatoid arthritis

\section{Introduction}

\subsection{Motivation}

Individuals use their hands every day to manipulate objects around them. After an individual grasps an object, they can squeeze, push, pull, or utilize any combination of those actions for manipulation. Previous studies have looked at forces required to grip objects and psychological factors that can affect grip $[4,12]$. Few studies have investigated how force is distributed throughout the hand when manipulating objects however. A pressure mapping system can provide quantitative data on hand force distributions to provide insight to the biomechanics of grip, push, and pull.

An estimated 1.3 million people in the United States have rheumatoid arthritis (RA) [8]. RA is an autoimmune disease that causes the immune system to attack the membrane lining joints. This attack leads to fluid filling the afflicted joints, which cause inflammation and pain. RA in the finger joints affects joint range of motion and can lead to decreased finger function. There are different treatment options for RA ranging from medication to corrective hand surgery [5]. The effectiveness of current treatment options is in dispute [1]. A pressure mapping system can provide quantitative data on the hand function of RA patients after different forms of treatment.

\subsection{Background}

Previous studies to investigate grasp have collected data using a grip dynamometer or a split cylinder [3, 6]. These devices provide a single force value to represent grip strength. However, this force value does not describe how force is distributed throughout the hand during grip. In recent years, with the advent of pressure mapping systems such as Fuji Film and Tekscan, researchers have used this technology to study grip $[10,11,13]$. The Tekscan pressure map-

*Corresponding author. E-mail: jwnich@umich.edu. Phone number: 989-964-1512. Address: 60107 Mile Road, Bay City, MI 48706 
ping system has been shown to be a reliable source to record force, pressure, and area data for a wide range of loads with proper calibration $[2,7]$.

Seo et al. (2007) and Welcome et al. (2004) both used a Tekscan pressure sensor to investigate the distribution of force throughout the hand when gripping an object. Seo et al. (2007) found that the fingertips and thenar region of the hand exert the highest normal force during the grip of a cylinder. Furthermore, they showed that force in the thenar region decreases as handle size increases. Welcome et al. (2004) also demonstrated that contact force is a function of handle diameter. They derived that an individual's grip force on a cylinder is a combination of grip and push forces.

\subsection{Hypotheses and Aims}

The goal of this study was to develop a better understanding of hand force distribution when gripping, pushing, and pulling a cylinder. For grip trials, we expected the fingertips and thenar region of the hand to have the highest concentration of normal force consistent with Seo et al. (2007). It is hypothesized that the phalanges and MCP joint line have the largest normal force concentration in pull trials and the thenar region have the majority of normal force concentration in push trials. Contact area is expected to increase as subjects exert more force on the cylinder and as subject hand area increases. It is also hypothesized that subjects with rheumatoid arthritis will exhibit a different force distribution during grip, push, and pull to compensate for their hand impairment.

\section{Methods}

\subsection{Subjects}

Ten subjects, six female and four male, with no hand impairment participated in this study with informed

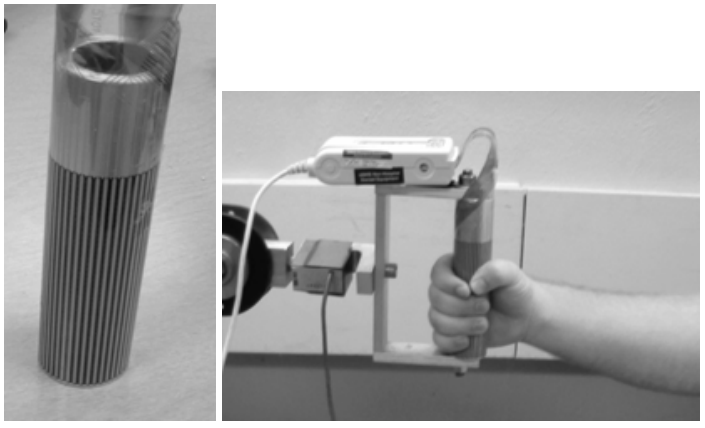

Figure 1. A. Pressure sensor. B. Push/pull testing apparatus

consent. Subjects were selected to represent the range of height found in the general population. Mean subject height was $172.8( \pm 8.1) \mathrm{cm}$. Anthropometric measurements, shown in Table 1 below, were taken of the subject's left and right hands.

The mean left hand length was $18.3( \pm 2.3) \mathrm{cm}$, mean left hand breadth was $8.0( \pm 0.9) \mathrm{cm}$, mean right hand length was $18.5( \pm 2.3) \mathrm{cm}$, and mean right hand breadth was $8.2( \pm 1.0) \mathrm{cm}$.

One female subject with rheumatoid arthritis was tested and is included as subject 11 . The subject had rheumatoid arthritis in both left and right finger joints and was being treated with medicine. The subject's left hand length was $17.6 \mathrm{~cm}$, left hand breadth was $8.3 \mathrm{~cm}$, right hand length was 17.5 , and right hand breadth was $7.7 \mathrm{~cm}$.

\subsection{Experiment Procedure}

At the beginning of the study, anthropometric measurements were recorded from each subject and pictures were taken of their hands. Subjects then performed a friction test to measure the friction force of skin on various surfaces. Friction force was measured for four different surfaces (aluminum, glass,

\begin{tabular}{|c|c|c|c|c|c|c|c|}
\hline & Gender & $\begin{array}{c}\text { Dominant } \\
\text { Hand }\end{array}$ & Height (cm) & $\begin{array}{c}\text { Left Hand } \\
\text { Leng th (cm) }\end{array}$ & $\begin{array}{c}\text { Left Hand } \\
\text { B readth }(\mathrm{cm})\end{array}$ & $\begin{array}{l}\text { Right Hand } \\
\text { Length }(\mathrm{cm})\end{array}$ & $\begin{array}{c}\text { Right Hand } \\
\text { Bread th }(\mathrm{cm})\end{array}$ \\
\hline Subject 1 & Male & Left & 171 & 18.0 & 8.8 & 18.4 & 8.7 \\
\hline Subject 2 & Male & Right & 179 & 21.6 & 8.8 & 21.9 & 9.0 \\
\hline Subject 3 & Female & Right & 173 & 18.5 & 8.1 & 18.7 & 8.8 \\
\hline Subject 4 & Male & Right & 183 & 22.7 & 9.6 & 22.8 & 10.1 \\
\hline Subject 5 & Female & Right & 161 & 17.2 & 7.6 & 16.5 & 7.7 \\
\hline Subject 6 & Female & Left & 179 & 16.1 & 7.3 & 16.9 & 7.5 \\
\hline Subject 7 & Female & Left & 165 & 16.3 & 8.1 & 16.4 & 8.2 \\
\hline Subject 8 & Female & Left & 167 & 16.5 & 6.8 & 16.8 & 7.1 \\
\hline Subject 9 & Female & Right & 166 & 16.5 & 6.6 & 17.0 & 6.8 \\
\hline Subject 10 & Male & Right & 184 & 19.4 & 8.4 & 19.2 & 8.2 \\
\hline Subject 11 & Female & Right & 163 & 17.6 & 8.3 & 17.9 & 8.6 \\
\hline
\end{tabular}


rubber, acrylic paint) according to the procedures outlined by Seo et al. (2005).

The surfaces were pulled with a strain gauge and force required to move the surface across the hand was recorded. Left and right hand grip strengths were measured using a Jamar grip dynamometer as an average of three trials. Two minutes of rest was provided between trials.

To collect contact area and force distribution data, a pressure sensor (Tekscan Sensor Model 5101) was wrapped around a $3.81 \mathrm{~cm}$ diameter aluminum cylinder, shown in Figure 1. Subjects performed a series of grip, push, and pull trials on the cylinder with the left and right hand. The cylinder was fastened to a secured handle for push and pull trials and was unconstrained for grip trials, Figure 1. A load cell was attached to the secured handle to record push/pull force data to compare with the force map data.

Subjects were seated with their upper arm vertical and their forearm horizontal. Trials were recorded at $100 \%, 50 \%$, and $25 \%$ maximum voluntary exertion for each hand. Three trials were performed at $100 \%$ maximum voluntary exertion and one trial for $50 \%$ and $25 \%$ exertions. Subjects were given two minutes of rest between each trial to minimize fatigue.

\subsection{Hand Pressure Map System}

The Tekscan I-Scan pressure mapping system was used to record force and contact area data, shown in Figure 3 below. This system uses a matrix sensor composed of two polyester sheets, conductive electrodes, and conductive ink to capture this data. The sensor used in this study measured $111.8 \mathrm{~mm} \times 111.8$ $\mathrm{mm}$ and contained 1,936 individual sensing elements, or sensels $\left(15.5\right.$ sensels $\left./ \mathrm{cm}^{2}\right)$. The electric signals from the sensor are processed by Tekscan software
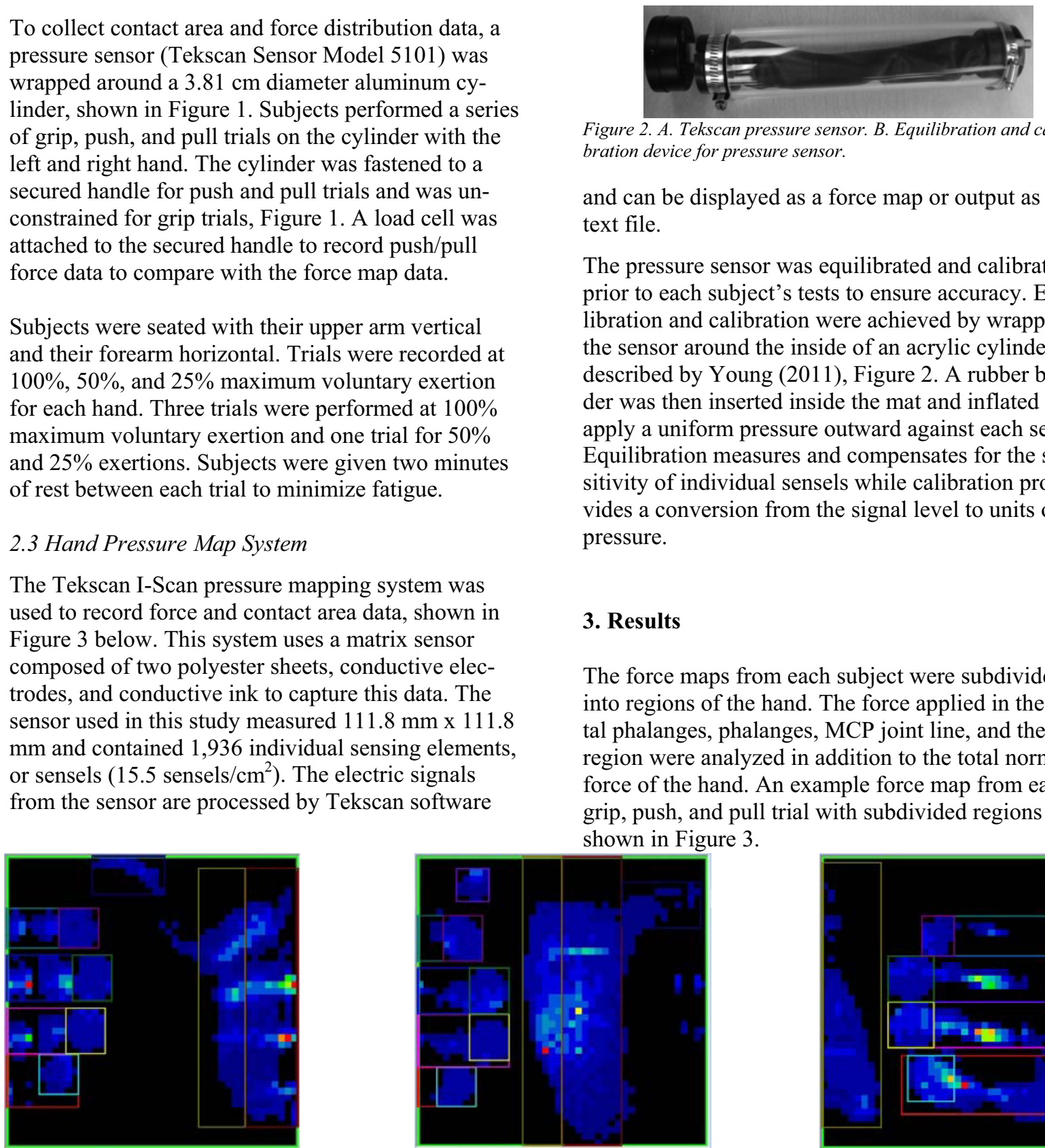

Figure 2. A. Tekscan pressure sensor. B. Equilibration and calibration device for pressure sensor.

and can be displayed as a force map or output as a text file.

The pressure sensor was equilibrated and calibrated prior to each subject's tests to ensure accuracy. Equilibration and calibration were achieved by wrapping the sensor around the inside of an acrylic cylinder as described by Young (2011), Figure 2. A rubber bladder was then inserted inside the mat and inflated to apply a uniform pressure outward against each sensel. Equilibration measures and compensates for the sensitivity of individual sensels while calibration provides a conversion from the signal level to units of pressure.

\section{Results}

The force maps from each subject were subdivided into regions of the hand. The force applied in the distal phalanges, phalanges, MCP joint line, and thenar region were analyzed in addition to the total normal force of the hand. An example force map from each grip, push, and pull trial with subdivided regions is shown in Figure 3.

Figure 3. Force maps collected from the pressure sensor using the Tekscan software. The force map on the left is from a grip trial, the force map in the middle is from a pull trial, and the force map on the right is from a push trial. Lighter colors are higher forces. 
Table 2. Grip strength measurements.

\begin{tabular}{lcccc}
\hline & $\begin{array}{c}\text { Left Hand Standard } \\
\text { Grip Strength (N) }\end{array}$ & $\begin{array}{l}\text { Right Hand Standard } \\
\text { Grip Strength (N) }\end{array}$ & $\begin{array}{l}\text { Left Pressure Map Total } \\
\text { Grip Surface Force (N) }\end{array}$ & $\begin{array}{c}\text { Right Pressure Map Total } \\
\text { Grip Surface Fo rce (N) }\end{array}$ \\
\hline Subject 1 & 461.11 & 467.04 & 290.20 & 929.86 \\
Subject 2 & 424.04 & 481.87 & 243.15 & 311.51 \\
Subject 3 & 296.53 & 330.63 & 522.51 & 519.22 \\
Subject 4 & & & 1047.95 & 610.27 \\
Subject 5 & 219.43 & 249.09 & 289.15 & 335.67 \\
Subject 6 & & & 598.98 & 174.32 \\
Subject 7 & 306.91 & 298.02 & 236.37 & 174.00 \\
Subject 8 & 326.19 & 323.22 & 207.24 & 156.02 \\
Subject 9 & 252.05 & 278.74 & 240.74 & 278.87 \\
Subject 10 & 419.59 & 498.18 & 267.34 & 369.75 \\
Subject 11 & 101.12 & 133.74 & & \\
\hline
\end{tabular}

\subsection{Comparison of standard grip strength to pressure mapped total grip exerted force.}

Grip strength values from the grip dynamometer are compared with grip surface force values measured by the pressure sensor in Table 2. The standard grip strength values for subjects 4 and 6 are absent from the table because there was not access to a grip dynamometer at their test location. Three subjects $(3,5$, 11) had higher grip force values on the pressure sensor while the rest of the subjects had higher grip force values on the grip dynamometer. Subject 1 is an exception with the left and right hand measuring higher grip forces on a different testing apparatus.

\subsection{Effect of increasing exertion force and increasing hand area on contact surface area.}

The average hand contact area during grip trials from the ten non-impaired subjects is shown in Figure 4 as a function of increasing exertion. The contact area of the hand increased by $48 \%$ from the $25 \%$ exertion trial to the $50 \%$ exertion trial and increased by $37 \%$ from the $50 \%$ exertion trial to the $100 \%$ exertion trial. The phalanges, MCP joint line, and thenar region all showed an increase in contact area as exertion increased.

The average hand contact area during 100\% exertion grip trials from the ten non-impaired subjects was analyzed as a function of increasing hand surface area. Hand surface area was defined as the product of hand length, measured from the tip of the middle distal phalange to the crease of the wrist, and hand breadth, measured from the outside of the metacarpal joint of the first phalange to the outside of the metacarpal joint of the fourth phalange. In general, as the hand surface area increased the hand contact area increased as well, shown in Figure 5. A regression equation was calculated for the whole hand contact area data as a function of hand surface area and was

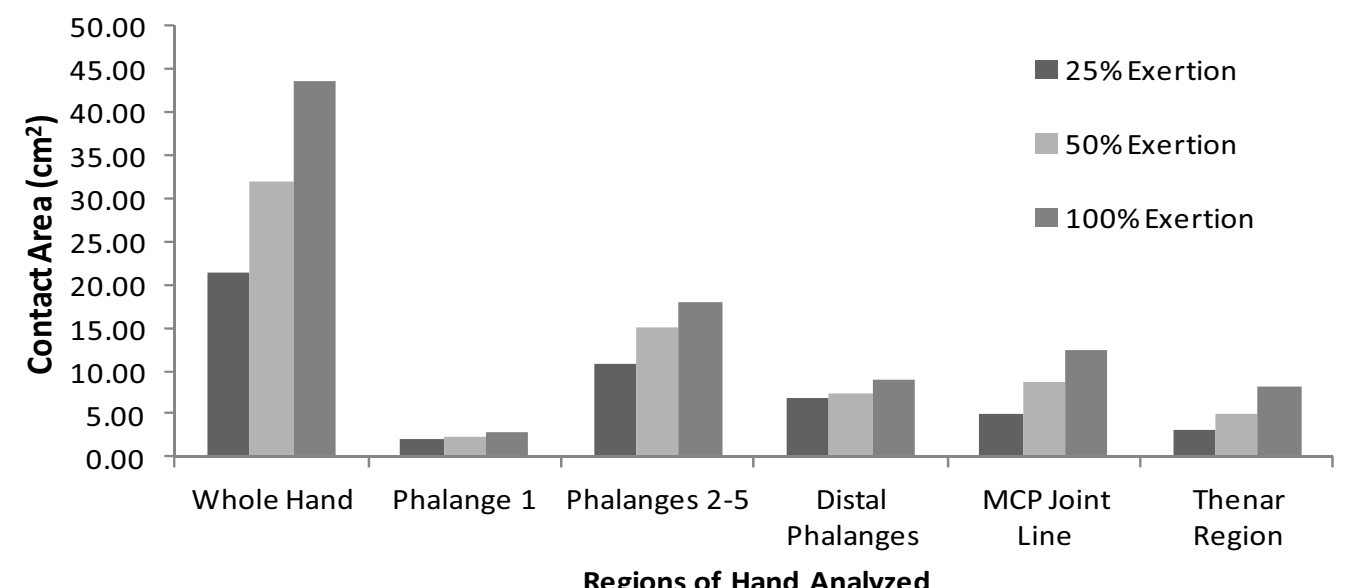

Figure 4. Hand contact area measured for varying levels of exertion during grip trials. Different regions of the hand were identified and contact area was calculated for each region. 


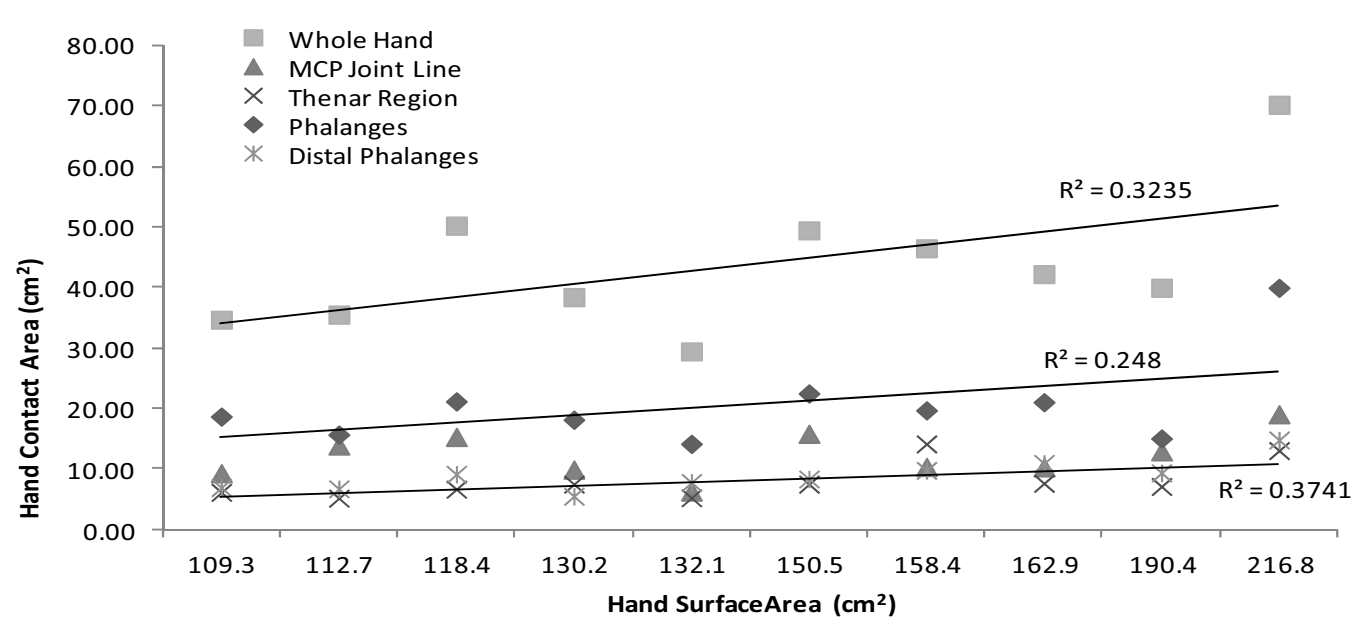

Figure 5. Hand contact area plotted as a function of hand surface area during grip trials. Regression equations were calculated for the whole hand, phalanges, and thenar region. $R^{2}$ values are shown on the graph.

found to have an $\mathrm{R}^{2}$ value of 0.3235 . There were un expected drops in contact area for several subjects given their measured hand area. Total contact area appears to be a function of gender also because males in general have a higher contact area than females.

\subsection{Relative distribution of force throughout hand.}

Relative force distributions throughout the hand were analyzed and compared for grip, push, and pull trials in Figure 6. The fingers, thumb, MCPs, and thenar region of the hand were identified in the force map from each trial. The phalanges were labeled 1-5, with phalange 1 as the thumb. Force distributions of each of these regions were calculated as percentages of the total applied normal force of the whole hand. There was an obvious increase in the amount of force applied in the MCP joint in the pull trials compared to the grip and push trials. Also, the relative amount of force applied in the palm in the push trial was $45 \%$ higher than both the grip and pull trials.

There is little variation in relative fraction of normal force in each region as exertion level increases. There are however distinct regions that exert a higher percentage of normal force in each different trial. The MCP joint line exerts a higher amount of relative force in pull trials compared to the push and grip trials. The palm applies a significant amount of force in the push trials compared to the other two trials.

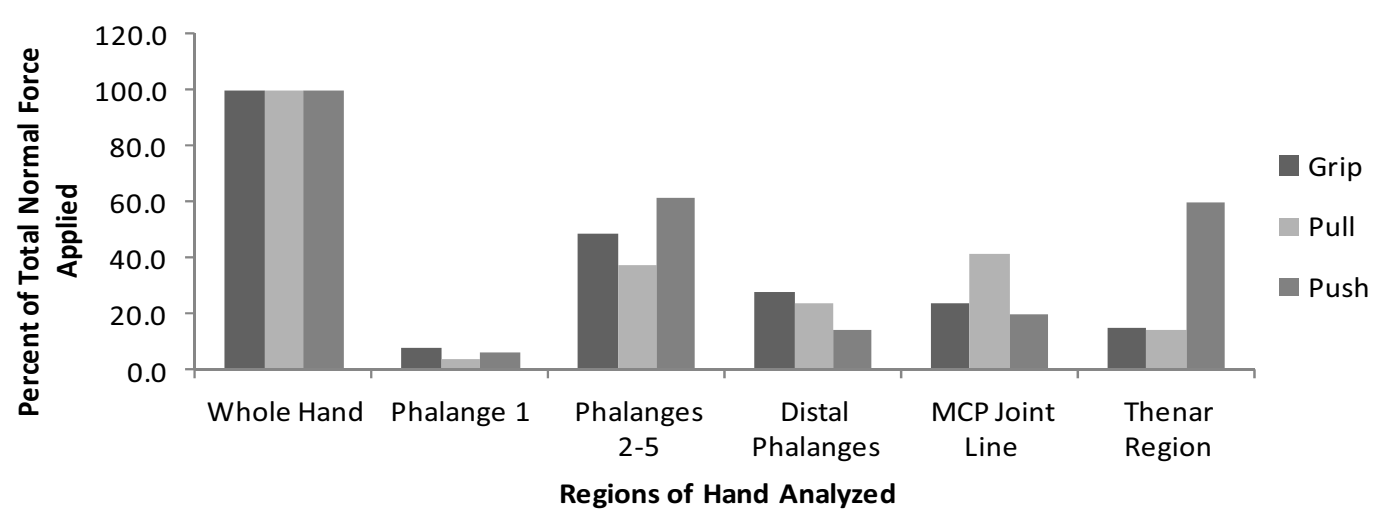

Figure 6. Forces measured in different regions of the hand as a relative percentage of the total normal force applied by the hand for grip, pull, and push trials. The data represents the average of all left hand data from the ten non-impaired subjects. 


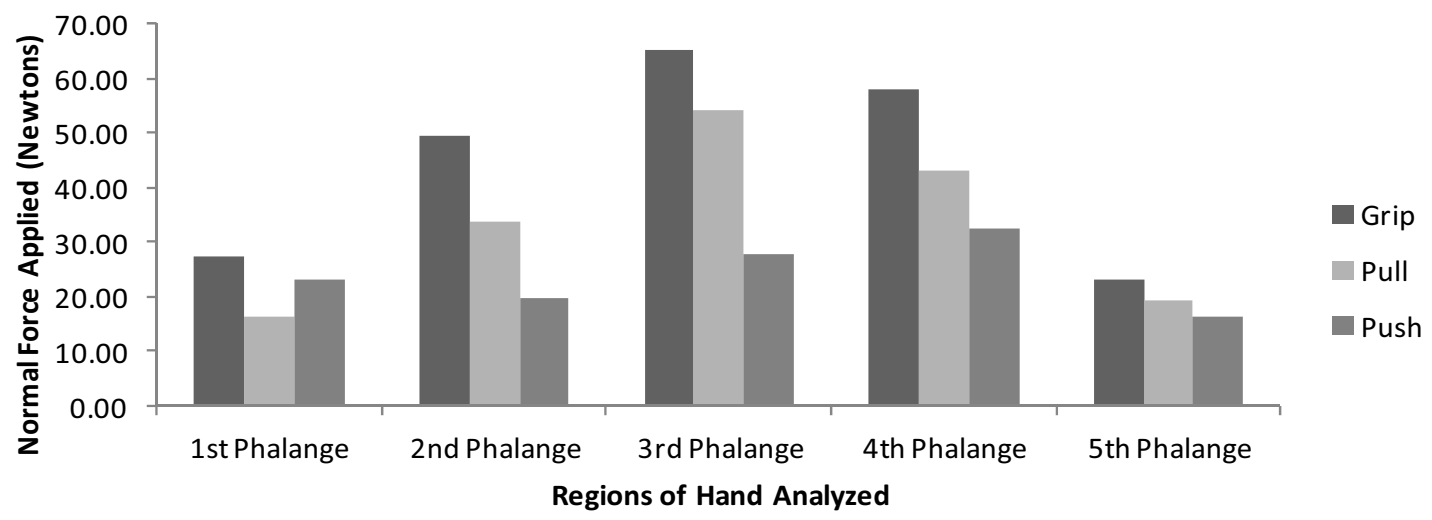

Figure 7. Forces measured in each phalange for grip, pull, and push trials. The data represents the average of all left hand data from the ten non-impaired subjects.

Figure 7 shows a breakdown of phalange forces for each trial type. The second, third, and fourth phalanges exerted the highest force for the grip and pull trials. All five phalanges exerted approximately the same amount of normal force in the push trial.

\subsection{Effect of rheumatoid arthritis}

Limited analysis was performed to compare the average force distribution from the ten non-impaired subjects and the force distribution from the one rheumatoid arthritis subject. The average non-impaired subject exerted approximately $40 \%$ more relative force in the phalanges than the RA subject during grip trials. The thumb and distal phalanges of the RA subject exerted a higher force relative to all the phalanges compared to the non-impaired subjects, however. In pull trials, the third and fourth phalanges exerted the highest force relative to the other phalanges. There was only a small amount of force registered on the pressure sensor during push trials. The phalange force that did register was located in the distal regions.

\section{Conclusion}

\subsection{Discussion}

This study investigated the different relationships between force and contact area during activities of grip, push, and pull. Resultant force data from the pressure sensor wrapped around the cylinder was compared with force data from the grip dynamometer. Overall, subjects had a higher measured grip strength on the dynamometer. The different force measurement between the two devices was possibly caused by their difference in geometry. The pressure sensor was wrapped around a cylinder which allowed subjects to have a higher contact area with the test surface compared to the handle of the dynamometer. A higher contact area on the gripping surface leads to a greater distribution of force, which causes a smaller resultant grip force for the pressure sensor. More trials need to be performed to confirm this relationship however.

The study also showed that increased exertion during grip, push, and pull led to a higher hand contact area with the underlying surface. Two mechanisms could be responsible for the increase in contact area. First, as exertion increases, the skin surface of the hand deforms. Second, sensels on the pressure sensor that previously did not register any force may register the 
increased exertion. The study also showed that increased hand area is associated with increased hand contact area. The relationship between hand area and hand contact area has not previously been defined, therefore it should not be assumed to be perfectly linear. The drops in hand contact area in Figure 5 were potentially caused by calibration and equilibration settings on the pressure sensor or hand measurement uncertainty. For example, the subject with the $132.1 \mathrm{~cm}^{2}$ hand area had a much lower contact area than the expected value. This subject was a small female and had a lack of measured data for the $25 \%$ exertion trials. A higher sensitivity calibration should be applied for small percentile subjects or subjects with low grip strength. The drops in hand contact area could also be caused by the uncertainty in hand area measurements. The hand area measurements were calculated on a computer to minimize these uncertainties however.

The force distribution throughout the hand was analyzed for grip, push, and pull trials. Force was relatively evenly distributed in grip trials. This is in contrast to the pull trials where the MCP joint line exerted the highest force and in the push trials where the thenar region dominated. The majority of grip force is distributed along the axis of highest applied normal force. It is interesting that the second, third, and fourth phalanges exert the highest relative phalange force in grip and pull trials. A possible explanation is the extra length of these phalanges provides additional surface area for force to be applied. The rheumatoid arthritis subject exerted more relative force in their thumb and distal phalange region compared to the average force exerted by the nonimpaired subjects. The swelling of the phalange joints in this subject from RA decreases range of motion which likely affects how much force can be applied through each phalange. The distal phalanges and thumb must not be affected to the same degree in this subject. This allowed the subject to compensate for the lack of function in the phalanges by applying more force in the thumb and distal regions of the phalanges in grip, push, and pull trials. This is reinforced in the push trials where the only force that registered in the phalanges was in the distal regions. The third and fourth phalanges were found to exert the highest relative phalange force in both the RA subject and non-impaired subjects. While the force magnitudes are different, this suggests that the mechanism for pulling was the same in all subjects.

\subsection{Limitations}

Each pressure sensor was required to be equilibrated and calibrated in an environment with uniform pressure. To satisfy this requirement, the pressure sensors were placed in an air tight acrylic tube. A portion of the pressure sensor was outside the tube because it needed to be connected to a computer to record data. As a result, the pressure sensor was squeezed between acrylic and a rubber seal, which caused wrinkling. The pressure sensor then experienced additional wrinkling when it was wrapped around the aluminum cylinder during trials. The wrinkling from these two events disrupted the transmission of force data from specific columns to the Tekscan software. If more than two columns of sensels were missing from a pressure sensor, it was replaced with a new sensor. A linear interpolation between neighboring sensels was performed to fill in missing data.

The pressure sensors used in this study for the ten subjects with no hand impairment were calibrated at pressures of 10 PSI and 30 PSI. From previous testing, these pressures created a calibration range that allowed the pressure sensors to capture grip, push, and pull data. When the smallest percentile female subjects' data was analyzed however, the number of loaded sensels was significantly smaller than what was expected from an individual with smaller hand area. For future studies, a pressure sensor should be calibrated at a lower pressure range for the smallest percentile subjects.

\subsection{Future work}

For the next phase of this study, additional small percentile females and males should be recruited. These subjects will use a pressure sensor with higher sensitivity to collect data more accurately and fill in missing gaps in the current study. Future studies should also investigate a larger population of subjects with varying hand area to strengthen or reject the initial results found in this paper.

Also, additional subjects with rheumatoid arthritis (RA) should be recruited. An effort should be made to sample RA patients with varying severity of the disease and before and after corrective surgery. 


\section{References}

[1] K.A. Alderman, K.C. Chung, H. Myra Kim, D.A. Fox, P.A. Ubel, Effectiveness of rheumatoid hand surgery: contrasting perceptions of hand surgeons and rheumatologists, Journal of Hand Surgery 28-1 (2003), 3-11.

[2] K.N. Bachus, A.L. DeMarco, K.T. Judd, D.S. Horwitz, D.S. Brodke, Measuring contact area, force, and pressure for bioengineering applications: using Fuji Film and Tekscan systems, Medical Engineering and Physics 28 (2006), 483-488.

[3] J.R. Blackwell, K.W. Kornatz, E.M. Health, Effect of grip span on maximal grip force and fatigue of flexor digitorum superficialis, Applied Ergonomics 30 (1998), 401-405.

[4] G. Buckingham, J.S. Cant, M.A. Goodale, Living in a material world: how visual cues to material properties affect the way we lift objects and perceive their weight, Journal of Neurophysiology 102-6 (2009), 3111-3118.

[5] K.C. Chung, Procedure 55: silicone metacarpophalangeal joint arthroplasty

[6] C.S. Edgren, R.G. Radwin, C.B. Irwin, Grip force vectors for varying handle diameters and hand sizes, Human Factors 462 (2004), 244-251.

[7] B.J. Fregly, W.G. Sawyer, Estimation of discretization of errors in contact pressure measurements, Journal of Biomechanics 36-4 (2003), 609-613.

[8] C.G. Helmick, D.T. Felson, R.C. Lawrence, S. Gabriel, R. Hirsch, C.K. Kwoh, M.H. Liang, H.M. Kremers, M.D. Mayes, P.A. Merkel, S.R. Pillemer, J.D. Reveille, J.H. Stone, Estimates of the prevalence of arthritis and other rheumatic conditions in the United States: Part I, Arthritis \& Rhuematism 58-1 (2008),

[9] N.J. Seo, T.J. Armstrong, P. Drinkaus, Methods to measure static coefficient of friction between hand and other materials. (2005) ASB $29^{\text {th }}$ Annual Meeting.

[10] N.J. Seo, T.J. Armstrong, J.A. Ashton-Miller, D.B. Chaffin, The effect of torque direction and cylindrical handle diameter on the coupling between the hand and a cylindrical handle, Journal of Biomechanics 40 (2007), 3236-3243.

[11] N.J. Seo, T.J. Armstrong, Investigation of grip force, normal force, contact area, hand size, and handle size for cylindrical handles, Journal of Human Factors and Ergonomics Society 50 (2008), 734-744.

[12] G.P. Slota, M.L. Latash, V.M. Zatsiorsky, Grip forces during object manipulation: experiment, mathematical model, and validation, Experimental Brain Research 213-1 (2011), 125139.

[13] D. Welcome, S. Rakheja, R. Dong, J.Z. Wu, A.W. Schopper, An investigation on the relationship between grip, push, and contact forces applied to a tool handle, International Journal of Industrial Ergonomics 24-6 (2004), 507-518.

[14] J.G. Young, Biomechanics of hand/handhold coupling and factors affecting the capacity to hang on, Ph.D. Dissertation, University of Michigan, 2011. 